\title{
User friendly tobacco barn heat controller for use by upcoming farmers
}

\author{
Elisha C Mabunda ${ }^{1}$ ), Cleophas D K Mutepfe ${ }^{2}$ \\ 1 and 2 Department of Electrical Engineering University of Zimbabwe P.O. Box MP167 Mt Pleasant, Harare
}

\begin{abstract}
This article describes a project that controls the tobacco barn temperature to specified levels. The major components of this controller include the temperature sensor, the processor and electronic switches. The PIC 16F876A is the heart of the controller, from which decisions are done upon receiving the status of the barn temperature via the LM35DZ sensor. The $\mu$ Processor makes adjustments by reducing or increasing hot air into the barn. The electronic power switches are realised from the triacs that are appropriately rated in terms of current and voltage handling.

The controller has a simple display composed of seven segment LED display. Further, the controller has flexibility in that the controls are done in software. If changes are to be made, this can be achieved through software, without affecting the hardware. The temperature can be gradually increased enhancing better curing rather than just setting a fixed temperature at once as is the case with traditional approach.
\end{abstract}

Keywords: controller, sensor, interrupts, analogue to digital converter, amplification, interfacing, switching, common anode, seven segment, decoder, regulator, initialization.

\section{INTRODUCTION}

Tobacco curing is a very important aspect in turning a green tobacco leaf into a golden leaf for the auction floors or for later manufacturing of various products. Although it is necessary to have a tobacco curing method which is more environmentally friendly, this project has been designed to be able to control the temperature of a tobacco barn that uses either electricity or the traditional heating methods that use coal or firewood. The design incorporates a microprocessor as the major component for its flexibility in setting the temperature parameters. It is driven by software hence many temperature settings could be effected without changes to the hardware. This controller can be adopted for other similar temperature sensitive applications such as incubators or green houses.

The design includes electronic power switching devices, known as the triac. Triacs have no moving parts hence the system is almost maintenance free, other than the regular cleaning of the panels and the housing.

\section{SENSOR DESIGN}

The temperature sensing circuit is designed around an LM35 precision centigrade temperature sensor. The LM35 series are precision integrated-circuit temperature sensors whose output voltage is linearly proportional to the Celsius (Centigrade) temperature [1]. The choice of this device is based on its temperature range which extend over $-55^{\mathrm{c}}$ to $+150^{\mathrm{c}}$, as this is adequate for the tobacco curing range of $+30^{\circ} \mathrm{C}$ to $+75^{\circ} \mathrm{C}$. Fig.1. shows the configuration of the sensor circuit, and a supply voltage of $12 \mathrm{~V}$.

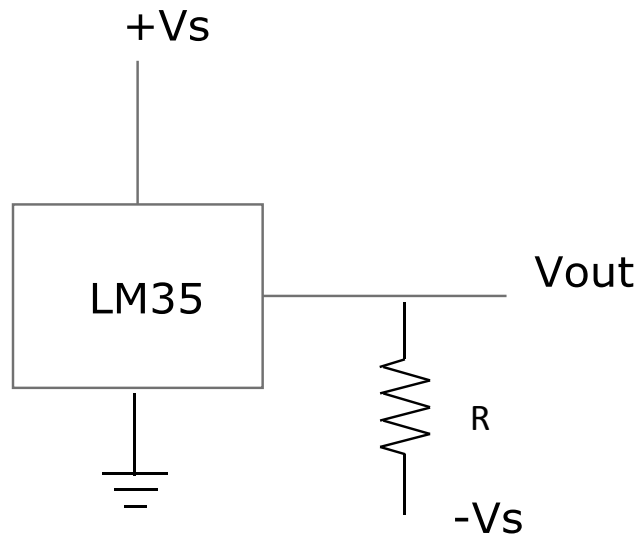

Fig.1 LM35 sensor configuration 
The value of $\mathrm{R}$ is given by Ohms Law i.e. $\mathrm{R}=\frac{V}{I}=\frac{12}{50.10-6}=2.4 \mathrm{k} \Omega$. Where $\mathrm{V}$ is the supply voltage and $\mathrm{I}$ is the specified current drain through the LM35 device. [1]

\subsection{LM35sensor testing.}

Selected sensor test results are shown in table 1.

Table1.

\begin{tabular}{|l|l|l|}
\hline Meter reading $(\mathrm{mV})$ & Corresponding Temperature $\left({ }^{0} \mathrm{C} / \mathrm{mV}\right)$ & $\begin{array}{l}\text { Temperature corresponding to Lab } \\
\text { Thermometer reading }{ }^{0} \mathrm{C}\end{array}$ \\
\hline 0.298 & 29.8 & 30 \\
\hline 0.361 & 36.1 & 36 \\
\hline 0.549 & 54.9 & 55 \\
\hline 0.751 & 75.1 & 75 \\
\hline
\end{tabular}

\subsection{Signal Amplification}

The signal from the sensor is too low to be used by the microprocessor, without amplification. The microprocessor requires a voltage of $5 \mathrm{~V}$ therefor the amplifier must have a gain of 5 . The gain of 5 is based on the maximum temperature of $100^{\circ} \mathrm{C}$; this maximum is bigger than $75^{\circ} \mathrm{C}$ the maximum for the tobacco burn (considering the worst case scenario), a voltage of $1 \mathrm{~V}$ is developed at the $\mathrm{V}$ out which then becomes $5 \mathrm{~V}$ after amplification.

To amplify the output of the sensor an LM358 chip is used. This chip has good gain and can operate on single rail of voltage range of 2 to $30 \mathrm{~V}$. The configuration of the amplifier is shown in Fig. 2. The gain is set by $\mathrm{R} 1$ and $\mathrm{R} 2$ to give the gain of 5 .

Gain $\mathrm{G}=\quad \frac{(R 1+R 2)}{R 1}=\frac{1.2+4.7}{1.2}=4.917[2]$ Retesting the sensor with the amplifier connected resulted in the data contained in table 2 that shows great improvement in the performance of the subsystem.

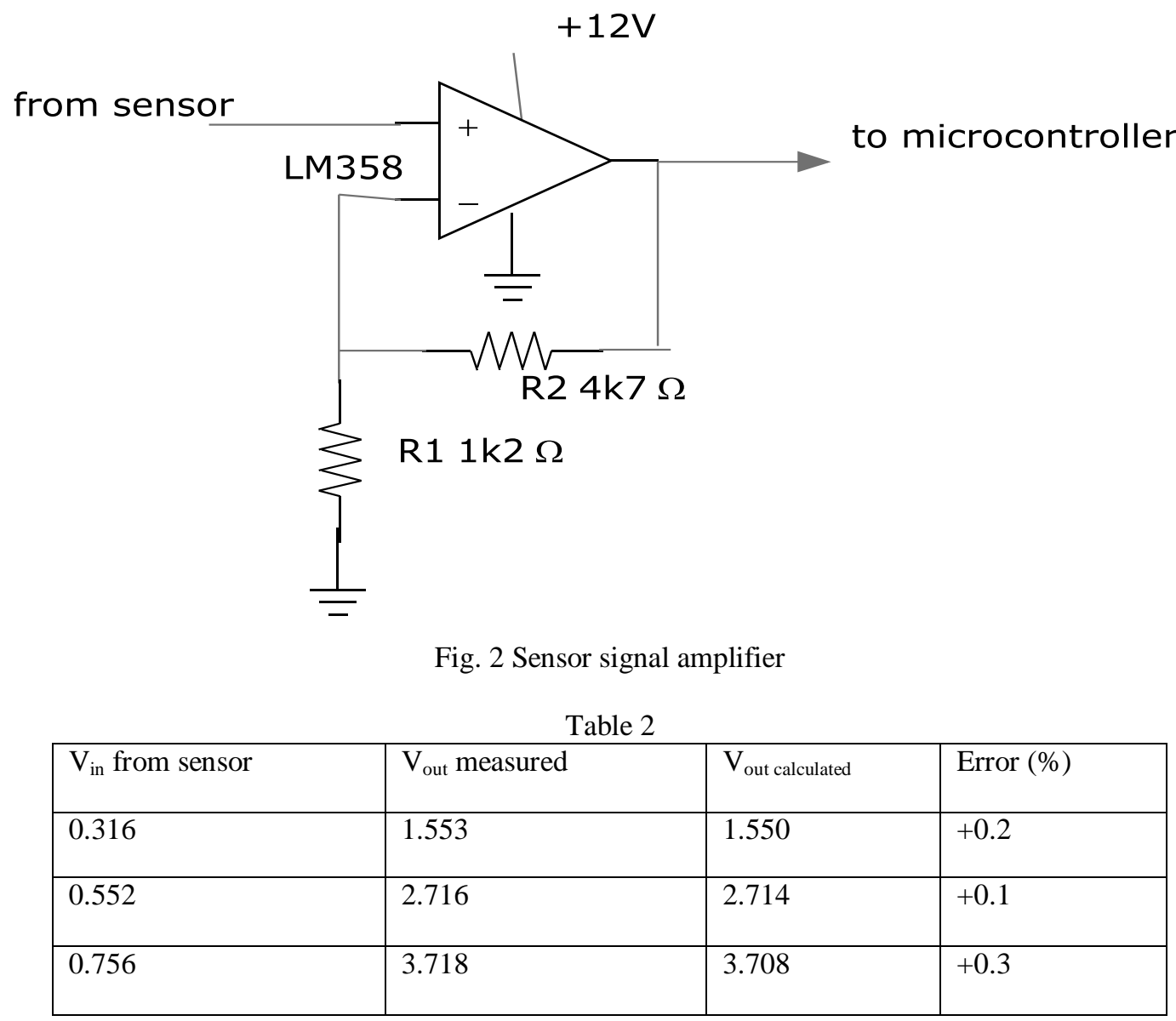




\section{THE POWER SECTION}

The triac was chosen to be the main switching device although two Silicon Controlled Rectifiers (SCR) connected back to back can also be used. In choosing the rating of the triac consideration has been given to the amount of load anticipated i.e. the heater power of 1000 watts and blower motor of 750 watts. Hence the triac is rated at $\frac{1000+750}{220}=7.95$ amps. Where 220 is the supply voltage and 7.95 is the resultant triac current rating. It is important to give the triac a margin of safety therefore the triac chosen is capable of handling up to $16 \mathrm{~A} 600 \mathrm{~V}$ which is a safety margin of the factor of 2 .

The interfacing of the triac section to the microcontroller is achieved via the Opt-coupler type MOC3041as shown in Fig. 3. The MOC3041 consists of gallium arsenide infrared emitting diodes optically coupled to a monolithic silicon detector performing the function of a Zero Voltage Crossing bilateral triac driver. [3]

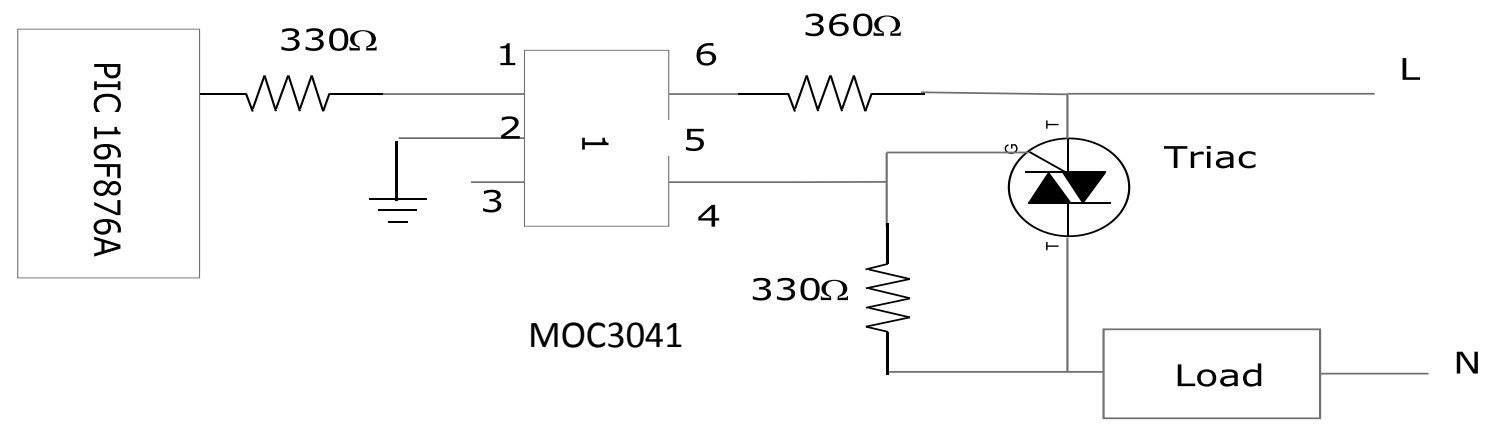

Fig.3 Microprocessor/Triac interfacing

IV.

THE DISPLAY UNIT

To make it possible to monitor the temperature and for the purposes of programming the system some kind of display is necessary. To certify this requirement the seven segment common anode LED display has been chosen for its merits. LEDs are much brighter compared to LCD and also easier to decode. A four digit display was assembled with the first bit pre-wired for the symbol for degrees Celsius. The decimal point after the second bit is also wired to provide tenths.

\subsection{The decoder}

To derive the seven segment display it is necessary to provide a decoder, for this an ICM7212AM decoder is implemented. This decoder is sufficient to cater for the bits that are required. The ICM7212AM can easily be configured by a software program and it also has a means of changing the brightness of the display depending on the distance from where it has to be viewed from. [3]

The LED seven segment displays are wired with each segment matched to the corresponding decoding pin of the decoder. A $5 \mathrm{~V}$ supply is connected to the common anodes and a $470 \Omega$ resistor connected to ground on one end and the other moved from decoding pin to the corresponding correct segment on the digits.

\section{THE MICROPROCESSOR (PIC16F876A)}

Implementing the controller using a Microprocessor PIC16F876A is made to simplify the necessary processes such as analogue to digital conversion and execution of the program that actuate heaters and blower to the tobacco burn.

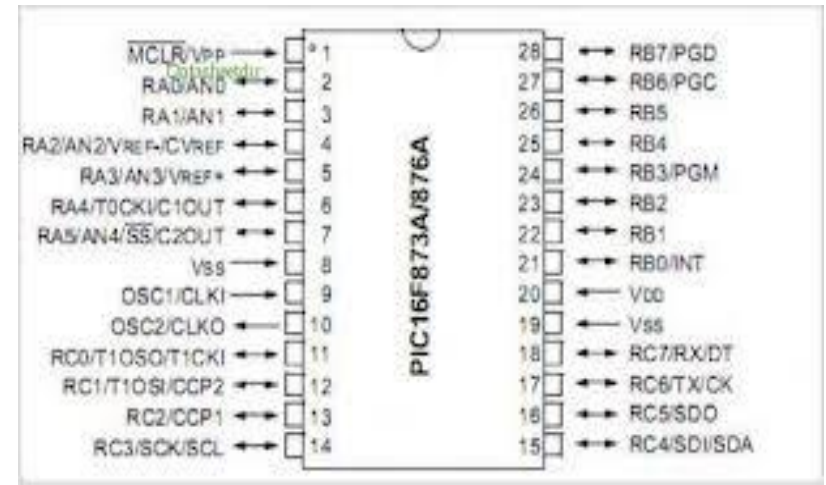

Fig. 4 PIC 16F876 microprocessor 


\section{THE COMPLETE SYSTEM}

Fig. 5 illustrates the overall temperature control system, with the PIC playing the major role in the execution of the code to perform the monitoring and control of the temperature in the pre-set levels as required by the crop. Since the temperature can be set at any level the system is useful for other application outside the tobacco crop.

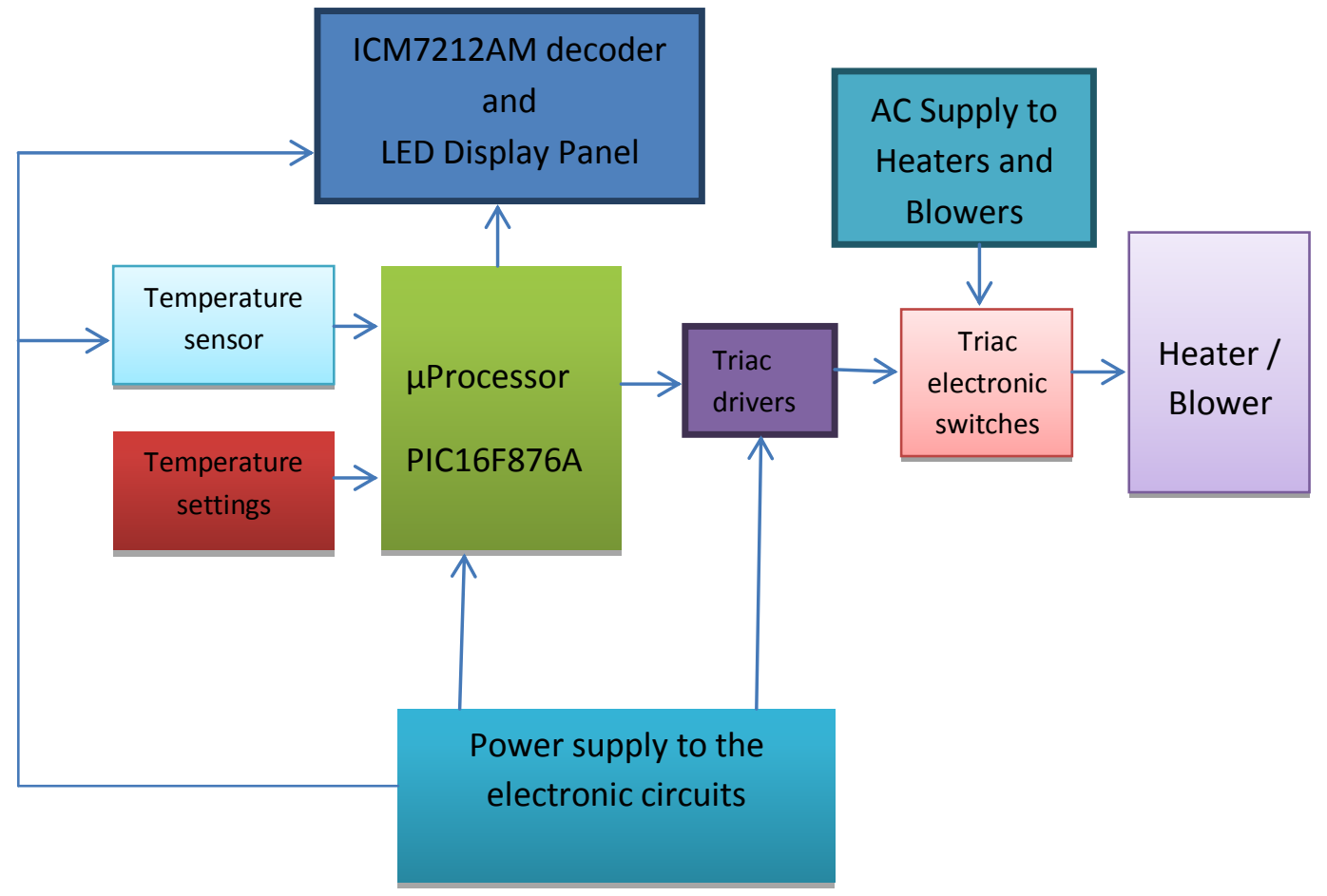

Fig.5. Block diagram of the complete system

VII. CODE DEVELOPMENT

The code development for this project is based on the assembly language for this type of PIC and the focused on four major sub-processes i.e. Temperature measuring and displaying, Temperature setting, Temperature controlling and Initialization.

\subsection{Temperature measuring and displaying}

After amplification the signal from the sensor is fed to the analogue input pin- RA0. An analogue to digital conversion (ADC) [4] is done using the ADC subroutine. In this process the Hex values are converted to binary coded decimal (BCD) by a HEX2BCD subroutine. The analogue to decimal result is loaded into registers for tenths, units and tens, which is then displayed as temperature on the seven segment displays.

\subsection{Temperature setting}

The present temperature is stored in registers PresetL and PresetH. The setting is either done by incrementing or decrementing these registers. This is achieved by interrupts which are initiated by pressing push buttons that are connected to pins RB4 and RB5. RB4 increases the temperature setting while RB5 decreases the same. To start setting a minimum, hold time of 5 seconds is allowed before setting can begin. This is necessary to avoid un-international temperature adjustments.

\subsection{Main Program}

The main program controls the whole system from power up, by calling the initialization that set up ports and subroutines and then call the start of ADC. After the ADC the program calls the compare subroutine to check if the value is within or above/below the pre-set values. The function then checks which flags have been set and then either increments or decrements the temperature by calling Increment_Temp or Decrement _Temp. The temperature is then displayed and the function goes to get the next ADC value. 


\section{CONCLUSIONS}

The implementation of this project provided a solid foundation that can be improved upon to come up with a more sophisticated design. However the objective of this particular design is to provide a simple solution that can be used by anyone who can read and is able to press a simple button. The design of this class has very minimal hardware components that make it very reliable. The code performed as expected albeit the need for tweaking to fine tune its execution as is the case with all software developments. The application of this design can be extended to incubators and green houses, to mention but a few.

\section{ACKNOWLEDGEMENTS}

Ndanga James an Electrical Engineering student at the University of Zimbabwe of the class of 2007 for helping with some of the research topics.

\section{REFERENCES}

[1] Texas Instruments Application notes

[2] Operational Amplifiers with Linear integrated circuits by William Stanley published by Charles E. Merril Publishing Company.

[3] Microprocessors Electronics book Series published by McGraw-Hill Inc.

[4] Analogue to Digital Conversion techniques by David F Hoeschele Jr. Published by John Wiley and Sons, Inc. 